\title{
The splice site variant rs11078928 may be associated with a genotype-dependent alteration in expression of GSDMB transcripts
}

Faer S Morrison', Jonathan M Locke', Andrew R Wood ${ }^{2}$, Marcus Tuke², Dorota Pasko², Anna Murray², Tim Frayling ${ }^{2}$ and Lorna W Harries ${ }^{1 *}$

\begin{abstract}
Background: Many genetic variants have been associated with susceptibility to complex traits by genome wide association studies (GWAS), but for most, causal genes and mechanisms of action have yet to be elucidated. Using bioinformatics, we identified index and proxy variants associated with autoimmune disease susceptibility, with the potential to affect splicing of candidate genes. PCR and sequence analysis of whole blood RNA samples from population controls was then carried out for the 8 most promising variants to determine the effect of genetic variation on splicing of target genes.
\end{abstract}

Results: We identified 31 splice site SNPs with the potential to affect splicing, and prioritised 8 to determine the effect of genotype on candidate gene splicing. We identified that variants rs11078928 and rs2014886 were associated with altered splicing of the GSDMB and TSFM genes respectively. rs 11078928 , present in the asthma and autoimmune disease susceptibility locus on chromosome 17q12-21, was associated with the production of a novel $\triangle$ exon5-8 transcript of the GSDMB gene, and a separate decrease in the percentage of transcripts with inclusion of exon 6, whereas the multiple sclerosis susceptibility variant rs2014886, was associated with an alternative TFSM transcript encompassing a short cryptic exon within intron 2.

Conclusions: Our findings demonstrate the utility of a bioinformatic approach in identification and prioritisation of genetic variants effecting splicing of their host genes, and suggest that rs11078928 and rs2014886 may affect the splicing of the GSDMB and TSFM genes respectively.

Keywords: GSDMB, Rs11078928, Asthma, Autoimmune disease, GWAS, SNP, Alternative mRNA splicing

\section{Background}

Genome wide association studies (GWAS) have greatly increased our understanding of the genetic basis of many complex diseases and traits by identifying single nucleotide polymorphisms (SNPs) that act as susceptibility factors for these diseases [1,2]. However, the results of GWAS only give us a genomic region associated with a particular disease or trait, and the causal SNP, gene and importantly, mechanism of action remain elusive. To date, only a handful of SNPs have been demonstrated

\footnotetext{
*Correspondence: L.W.Harries@exeter.ac.uk

'RNA mediated mechanisms of disease group, University of Exeter Medical School, EX2 5DW Exeter, UK

Full list of author information is available at the end of the article
}

as causal for a particular disease and the mechanism whereby the SNP confers disease risk identified. For example, GWAS has identified hundreds of loci associated with susceptibility to inflammatory or autoimmune diseases such as Crohn's disease, asthma, multiple sclerosis (MS) and type 1 diabetes (T1D), furthering our understanding of the genetic basis of these diseases and identifying areas of the genome for further study [3]. However, apart from a variant in the immunity-related GTPase family, M (IRGM) gene, which has been shown to confer susceptibility to Crohn's disease by altering miRNA binding at the site of the SNP [4], the causal genes and mechanisms in most cases remain to be identified. 
The slow rate of progress in converting advances made by GWAS into a fuller understanding of which genes are associated with disease, and their mechanistic actions, could be a reflection of the focus on protein coding variants, whereas most SNPs are in fact within non-coding regions of the gene [5,6]. At least $80 \%$ of the human genome has now been demonstrated to contain important regulatory sequences such as enhancers, silencers, small RNA binding sites and chromatin modifiers [7-12]. An important mechanism of gene regulation, alternative splicing, is a complex process requiring over 100 splicing factors that recognise regulatory elements such as exonic and intronic splicing enhancers and silencers (ESEs, ESSs, ISEs, ISSs), which bind trans-acting splicing regulatory factors [13] that bind to the mRNA secondary structure [14]. Regulatory elements include the splice acceptor AG, the polypyrimidine tract, the splice donor GT and the branch site adenosine residue [13]. In addition disruption of any of these sequences has the potential to alter splicing by re-directing the spliceosome or by altering binding of auxiliary factors to exonic and intronic splicing enhancers and silencers.

In this study, our aim was to follow a bioinformatic pipeline in order to identify autoimmune diseaseassociated variants with the potential to effect splicing, and to follow up interesting candidate variants by mRNA analysis in whole blood, an appropriate tissue for the analysis of autoimmune or inflammatory disorders. In this article, we report two variants in two genes that exhibit a genotype-associated effect on mRNA processing of their host genes.

\section{Results}

SNPs associated with autoimmune or inflammatory diseases are enriched for splice site variants

We performed a series of bioinformatic analyses to identify genetic variants that could potentially alter splicing of their host genes (Figure 1). 338 SNPs associated with one or more autoimmune diseases or inflammatory traits were identified that reached genome wide significance, and 7322 proxies were identified to these index SNPs. Of these, 31 SNPs were annotated as 'splice_region_variant' using the Biomart program (Ensembl; http://www. ensembl.org). When compared to 1000 sets of 338 random SNPs and their proxies, we found that $0.41 \%$ variants associated with autoimmune or inflammatory phenotypes were located in splice regions, compared with a mean of $0.28 \%$ for randomly selected variants (one tailed t-test $\mathrm{p}=0.028$; Figure 2), indicating that inflammatory or autoimmune SNPS were enriched for splice site variants.

\section{Two SNPs in splice site regions were shown to produce alternative splice products}

8 variants were prioritised for further analysis on the basis that they had the potential to create cryptic splice sites, interrupt polypyrimidine tract sequences or disrupt regulatory elements involved in splicing. The 8 variants prioritised for further analysis using this pipeline are shown in Table 1. Unusual bands were identified for half of the variants tested upon RT-PCR (rs11078928 (GSDMB), rs2014886 (TSFM), rs1260326 (GCKR) and rs3764021 (CLEC2D). Two bands (GCKR, CLEC2D) were artifactual, and the remaining two (GSDMB, TSFM) were further characterized and sequenced. We found no

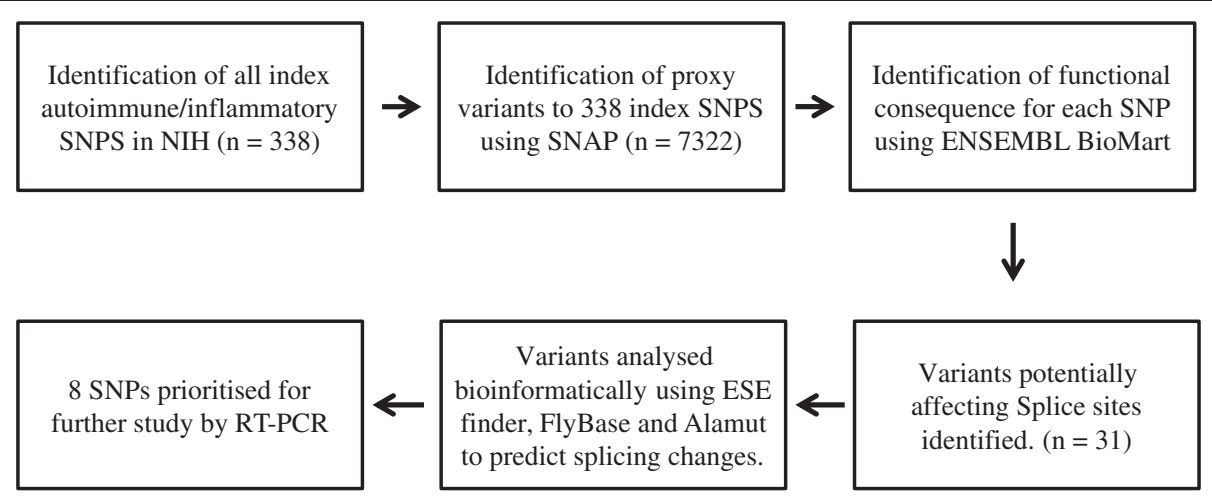

Figure 1 Bioinformatic pipeline used to predict splice site SNPs that are associated with autoimmune diseases and inflammatory traits. Index inflammatory SNPs were identified that had been associated by GWAS with susceptibility to autoimmune diseases and inflammatory traits. The proxies to these GWAS SNPs were pulled using the SNAP Proxy Search tool (Broad Institute). The 'functional consequence to transcript' for each SNP was identified as being 'Splice Site' or 'Essential Splice Site' using the Biomart function of Ensembl. These SNPs were then bioinformatically analysed to predict whether a splicing change was likely to occur, using the programmes ESE finder (web-based tool that predicts ESE element sequences that are bound by SR proteins), NNSplice (web-based tool that algorithmically predicts core spice site sequences in a given sequence) and Alamut (splicing mutation prediction programme that amalgamates predictions from five different splice site prediction algorithms to identify potential core splice sites in a given gene transcript). A subset of SNPs was then prioritised for further analysis of their splicing by RT-PCR. The numbers in brackets denote how many SNPs were identified at each stage. 


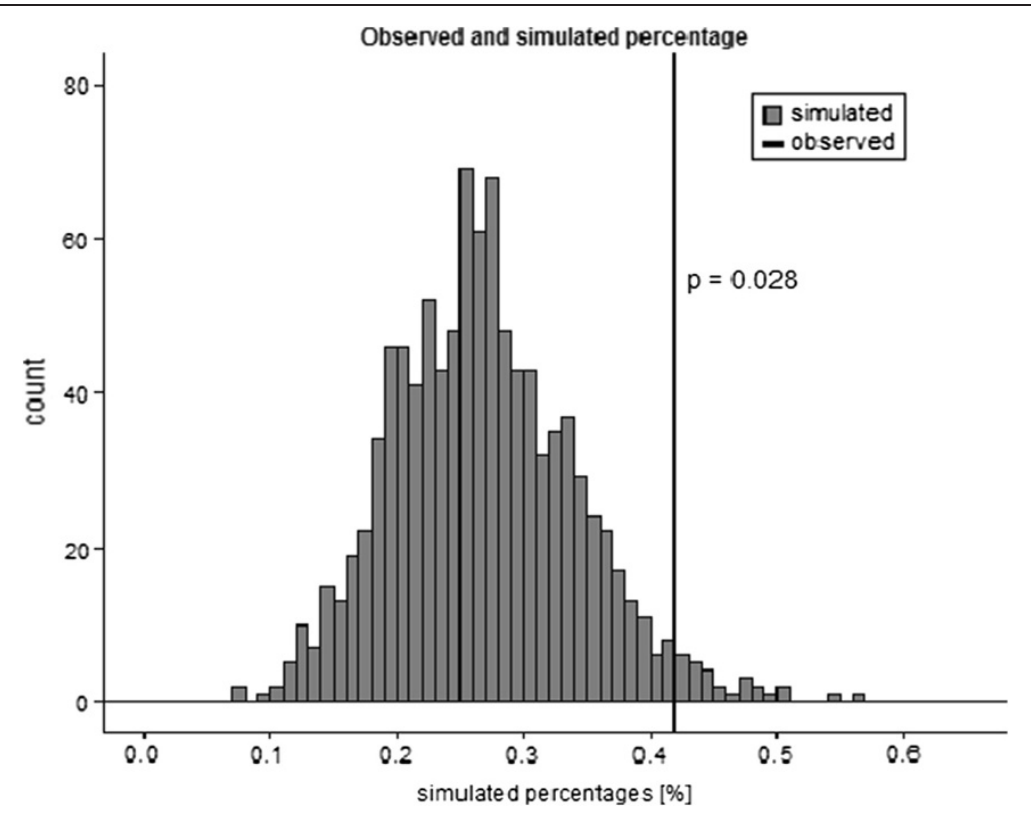

Figure 2 Enrichment of variants associated with inflammatory or autoimmune phenotypes in splicing control regions. We assessed the number of randomly selected variants located in splicing control elements by chance. For each set of 338 'index' SNPs and their proxies, the percentage of variants located in 'splice region elements' is given on the X-axis (simulated percentage), and the number of SNPs at each percentage (count) is given on the Y-axis. The observed number of autoimmune or inflammatory SNPs located in 'splice region elements' is given by the line.

evidence of altered splicing caused by the remaining variants, although we cannot rule out splicing changes not identified because of primer placement or transcript levels.

\section{Rs11078928 causes two separate splicing changes in the Gasdermin B (GSDMB) gene}

We found two separate splicing changes associated with the variant rs11078928 in the gene GSDMB. These changes include production of a novel transcript of GSDMB and a change in isoform ratio. The novel band associated with rs11078928 was found upon sequence characterisation to be a large deletion product lacking exons 5-8 of the gene GSDMB (Figure $3 \mathrm{~A}$ and Additional file 1). To determine whether this deletion was associated with genotype and therefore caused by the variant rs11078928, we designed Taqman assays to the wild-type and novel transcripts as described above from the custom assay service available from Life Technologies (Life Technologies, Foster City, USA). We found evidence to suggest a genotype-associated effect on the expression levels of this deletion product; with homozygotes for the major (A) allele, showing increased expression of this alternate $\Delta 5-8$ transcript, and homozygotes for the minor (G) allele showing negligible expression of the transcript compared with heterozygous individuals or those homozygous for the A allele $(\mathrm{p}=0.0001$; Figure 3B).
Moreover, four reference transcript sequences are described for this gene in expressed sequence tag (EST) databases; two transcripts (NM_001165958.1 and NM_001165959.1) include exon 6, whilst this exon is deleted in the remaining two (NM_001042471.1 and NM_018530.2). Therefore, we quantified relative expression of these different isoforms according to genotype using Taqman Assays (Life Technologies, Foster City, USA) (Additional file 2). Although there was no significant change in expression of the isoforms lacking exon 6 , we found that the isoforms which include exon 6 have almost no expression in homozygotes for the minor allele of rs11078928 compared with heterozygous individuals, and those homozygous for the $\mathrm{A}$ allele $(p=0.0002$; Figure $3 C)$. This striking genotypespecific expression difference suggests that rs11078928 or an associated variant may be altering exon 6 inclusion in the GSDMB transcript. The Alamut Mutation Interpretation Software (Interactive Biosoftware, Rouen, France) predicted that rs11078928 would result in deletion of exon 6. We also quantified expression of the WT transcript and overall expression of GSDMB, to determine whether these were altered by genotype. The expression differences between genotypes showed decreased expression for those individuals carrying the minor allele, although they did not reach statistical significance (results shown in Figure 3D-E). 
Table 1 The 8 splice site SNPs prioritised for further analysis after following the bioinformatic pipeline set out

\begin{tabular}{|c|c|c|c|c|c|c|c|c|c|}
\hline $\begin{array}{l}\text { Proxy } \\
\text { (i.e. splice site SNP) }\end{array}$ & $\begin{array}{l}\text { GWAS variants in } \\
\text { LD with splice site } \\
\text { SNPs }\end{array}$ & $P$ value & $\begin{array}{l}\text { Phenotype } \\
\text { (risk allele) }\end{array}$ & PID & $r^{2}$ & $\begin{array}{l}\text { Host gene } \\
\text { of splice } \\
\text { site SNP } \\
\end{array}$ & $\begin{array}{l}\text { Alleles for splice } \\
\text { site SNP }\end{array}$ & $\begin{array}{l}\text { Consequence } \\
\text { to transcript }\end{array}$ & Prediction/location \\
\hline \multirow[t]{5}{*}{ rs11078928 } & rs 2872507 & $5 \times 10^{-11}$ & Ulcerative colitis (A) & 21297633 & 1 & GSDMB & $A>G$ & $\begin{array}{c}\text { Essential Splice } \\
\text { Site }\end{array}$ & $\begin{array}{l}\text { Destroys AG of acceptor splice site intron } \\
\text { 5: Predicted skip of exon } 6 \text { (Alamut) }\end{array}$ \\
\hline & rs2872507 & $5 \times 10^{-9}$ & Crohn's disease (A) & 18587394 & 1 & & & & \\
\hline & rs9303277 & $2 \times 10^{-9}$ & $\begin{array}{l}\text { Primary biliary cirrhosis } \\
(\mathrm{T})\end{array}$ & 20639880 & 0.905 & & & & \\
\hline & rs 2290400 & $6 \times 10^{-13}$ & Type 1 diabetes (?) & 19430480 & 0.905 & & & & \\
\hline & rs7216389 & $9 \times 10^{-11}$ & (Asthma (T)) & 17611496 & 0.905 & & & & \\
\hline rs2014886 & rs703842 & $5 \times 10^{-11}$ & multiple sclerosis (A) & 19525955 & 0.964 & TSFM & $C>T$ & $\begin{array}{l}\text { Essential Splice } \\
\text { Site }\end{array}$ & $\begin{array}{l}\text { Introduces cryptic donor splice site intron } \\
\text { 2: Likely to result in alternative exon inclusion }\end{array}$ \\
\hline \multirow[t]{2}{*}{ rs1260326 } & rs780094 & $7 \times 10^{-15}$ & C-reactive protein (A) & 18439548 & 0.933 & GCKR & $\mathrm{T}>\mathrm{C}$ & Splice Site & $\begin{array}{l}1 \text { bp from GT of splice donor exon 15, alters } \\
\text { splice site score }\end{array}$ \\
\hline & rs780093 & $5 \times 10^{-11}$ & Crohn's disease $(T)$ & 21102463 & 0.901 & & & & \\
\hline rs10263341 & rs886774 & $3 \times 10^{-8}$ & Ulcerative colitis (G) & 19915572 & 0.803 & DLD & $\mathrm{T}>\mathrm{C}$ & Splice Site & May interrupt polpyrimidine tract intron 6 \\
\hline rs1322077 & rs2301436 & $1 \times 10^{-12}$ & Crohn's disease $(T)$ & 18587394 & 0.904 & FGFR1OP & $\mathrm{T}>\mathrm{C}$ & Splice Site & May interrupt polpyrimidine tract intron 5 \\
\hline rs2020854 & rs2066808 & $1 \times 10^{-9}$ & Psoriasis (A) & 19169254 & 1 & STAT2 & $A>G$ & Splice Site & May introduce cryptic donor site intron 14 \\
\hline \multirow[t]{2}{*}{ rs55719896 } & rs8049439 & $2 \times 10^{-9}$ & $\begin{array}{l}\text { Inflammatory bowel } \\
\text { disease, early onset }(G)\end{array}$ & 19915574 & 0.965 & ATXN2L & $G>A$ & Splice Site & $\begin{array}{c}\text { Destroys AG of cryptic acceptor splice site } \\
\text { intron } 20\end{array}$ \\
\hline & rs4788084 & $3 \times 10^{-13}$ & Type 1 diabetes (G) & 19430480 & 0.824 & & & & \\
\hline rs3764021 & rs3764021 & $5 \times 10^{-8}$ & Type 1 diabetes $(\mathrm{G})$ & 17554300 & 1 & CLEC2D & $C>T$ & Splice Site & $\begin{array}{c}\text { May introduce cryptic donor splice site } \\
\text { exon } 2\end{array}$ \\
\hline
\end{tabular}

The table shows the 8 SNPs that lie within splice sites of the genes listed. The index GWAS autoimmune/ inflammatory SNPs that are in LD $\left(r^{2}<0.8\right)$ with the splice site SNPs are shown, as well as the splice site prediction/location. PID PubMed Accession, LD linkage disequilibrium. 


\section{A}

\section{GSDMB WT isoforms}

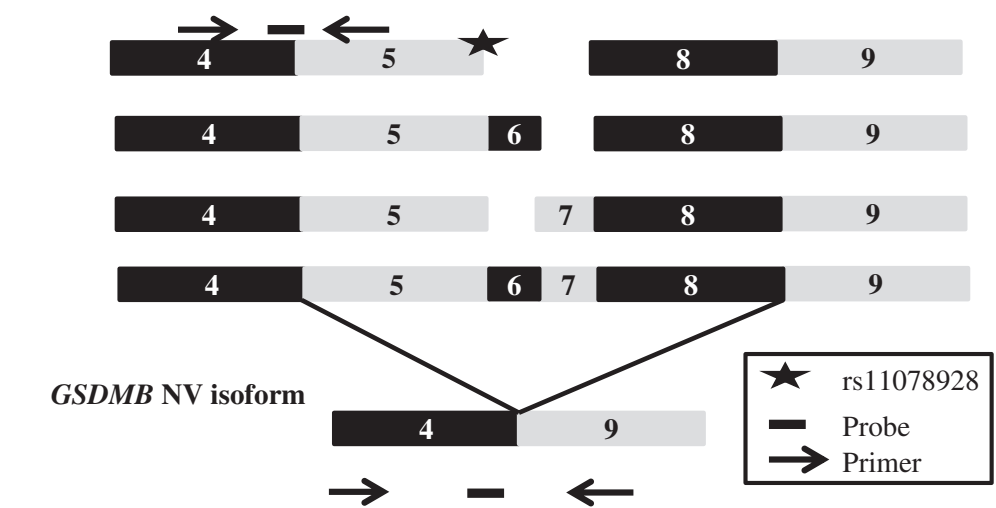

B

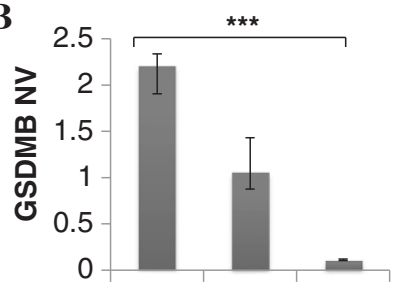

AA AG GG

D

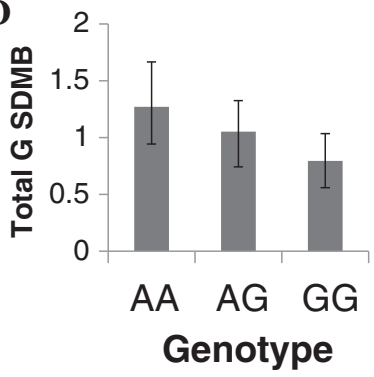

C

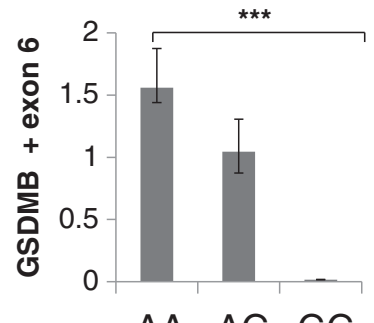

AA AG GG

$\mathbf{E}$

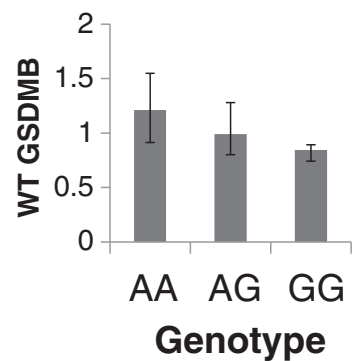

Figure 3 Wild-type (WT) and Novel Variant (NV) isoforms of the GSDMB gene and their expression with genotype. A. Showing exons 4-9 of the four RefSeq isoforms and the novel GSDMB transcipt (GSDMB NV), which is missing exons 5-8. Half the Reference Sequence (WT) transcripts include exon 6 and two are lacking exon 6; NM001165958.1 is the full length transcript. The star shows the position of rs 11078928 (acceptor splice site of intron 5). The location of the Custom Taqman Assay Primers (indicated by an arrow) and probe (indicated by a rectangle) are shown. B. Chart showing the expression of the novel transcript GSDMB NV by genotype. Homozygotes for the minor allele show negligible expression of the novel transcript. Expression is normalised to the endogenous control RPLPO, and is shown relative to the expression of GSDMB NV in heterozygotes. Significant results $(P<0.05)$ are indicated by an asterix. $C$. Chart showing the expression with genotype of the GSDMB transcripts which include exon 6 (NM001165958.1, NM001165959.1). Homozygotes for the minor allele show no expression of exon 6-containing transcripts. Expression is normalised to the endogenous control RPLPO, and is shown relative to the expression of exon 6 GSDMB transcripts in heterozygotes. D-E. Charts showing total expression of GSDMB (all RefSeq isoforms, including novel) and expression of GSDMB WT (all RefSeq isoforms). Expression is normalized to the endogenous control RPLPO, and is shown relative to total and WT expression respectively in heterozygotes. Both show a decrease in expression with the minor allele, although these results did not reach statistical significance.

Rs2014886 creates a cryptic donor splice site and alters splicing of the Ts translation elongation factor, mitochondrial (TSFM) gene.

We identified a novel band in carriers of the ' $\mathrm{T}$ ' allele for rs2014886 which we were unable to isolate by conventional means due to the large size of the product. The size of the band ( $530 \mathrm{bp}$ ) corresponded to a TSFM transcript with the inclusion of a short exon, where the donor site is created by rs2014886 and which has EST (expressed sequence tag) evidence of use [15], but which is not present in the reference sequence (RefSeq) transcripts. This donor site is preceded by a strong acceptor site that is present $38 \mathrm{bp}$ upstream of the variant, which also has evidence of use in EST databases [15] (Figure 4A and Additional file 3). We therefore designed RT-PCR primers specific to the predicted novel transcript, to 


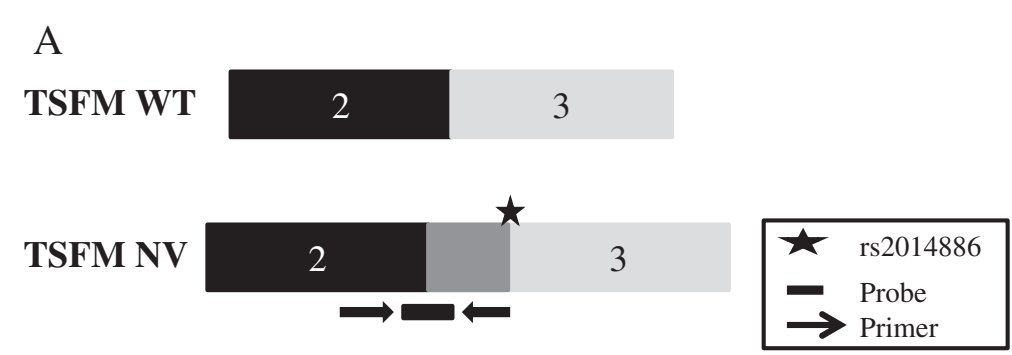

B

TSFM MT

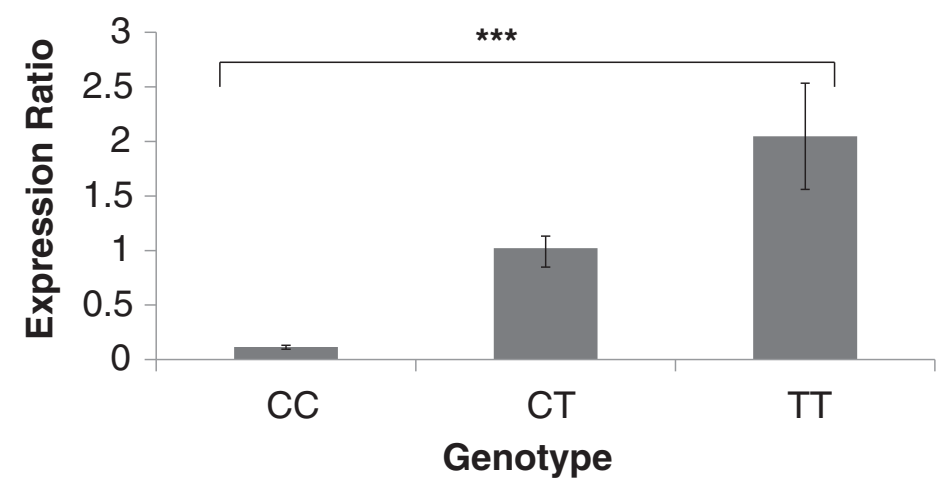

Figure 4 WT and NV isoforms of TSFM and their expression with genotype. A. Showing exons 2-3 of the RefSeq transcript (there are four RefSeq isoforms for TSFM, however, they are identical over this region of the gene) and the novel TSFM transcript (TSFM NV), which has an alternate $38 \mathrm{bp}$ exon included between introns 2 and 3. The star shows the relative position of rs2014886 (introducing an intronic donor splice site within intron 2. B. Chart showing the expression of the novel transcript TSFM NV by genotype. Homozygotes for the major allele show negligible expression of the novel transcript. Expression is normalised to the endogenous control RPLPO, and is shown relative to the expression of TSFM NV in heterozygotes. Statistical significance is indicated by an asterix.

confirm its identity. By this method, we were able to sequence the product and verify that the transcript includes a short 38 bp exon insertion within intron 2 of TSFM (Figure 4A). Expression of the novel transcript was found to be associated with genotype, with individuals carrying two minor ( $\mathrm{T}$ ) alleles expressing the novel transcript at levels approximately 2 -fold higher than individuals carrying two major (C) alleles $(\mathrm{p}=0.00005$; Figure 4B). However, we found no significant differences in expression between individuals of different genotypes for expression of total TSFM and TSFM WT.

\section{Discussion}

Although hundreds of SNPs have been associated with autoimmune diseases by GWAS [1,2] the causal gene and mechanism in most cases remains elusive. We prioritised 8 variants with bioinformatic evidence to alter splicing by creating cryptic splice sites, interrupting polypyrimidine tract sequences or disrupting regulatory elements involved in splicing. Here we provide evidence that a bioinformatic pipeline may be followed in order to identify and prioritise variants that are likely to affect splicing of their host gene. We have identified a genotype-associated effect on alternative splicing for two of the 8 SNPs (rs11078928 and rs2014886) on the processing of the GSDMB and TSFM genes respectively.

Variant rs11078928 is associated with two separate splicing changes in the gene GSDMB, which could possibly be of functional significance, whereas, the effect of rs2014886, within TSHM most likely has little functional consequence, since it is not affected by genotype. For the remaining 6 SNPs, we found no evidence for altered splicing. This could indicate that the SNPs in or near these splice sites were too weak to re-direct the splicing machinery, although it cannot be overlooked that the sensitivity of the assays may have affected identification of splice variants (especially at low levels of expression). Alternatively, these genes may be differentially expressed in a tissue other than blood. Nevertheless, variation in splicing regulatory sequences leading to aberrant splicing is a relatively common occurrence and may explain some of the signals identified by GWAS [16].

Variant rs2014886, a C > T change in intron 2 of the TSFM gene, was predicted to create a cryptic donor splice site within the intron, and transcripts derived from the use of this cryptic splice site, 38 bp downstream of a strong acceptor splice site, are represented in EST databases [15]. We confirmed that the minor allele of the SNP leads to the production of this alternative 
splice product, which includes the 38 bp short exon within intron 2. Negligible amounts of the insertion product (referred to as TSFM NV) were expressed from the transcripts carrying the major $(C)$ allele (Figure $4 \mathrm{~B}$ ). The difference in age between genotypes in our cohort for the variant rs2014886 reached statistical significance, so it is possible that the effects we note for TSFM splicing could be driven by differences in mean age rather than genotype. The insertion of the novel $38 \mathrm{bp}$ intron causes a frameshift event leading to the generation of a premature termination codon at a position 85 bp downstream of the insertion. This would in all probability render the novel transcript susceptible to the nonsensemediated decay mRNA surveillance pathway [17]. TSFM has been identified as a likely candidate gene in multiple sclerosis susceptibility [18], and its expressio14, para $2 \mathrm{n}$ was recently found to be correlated with a variant that alters an enhancer region [19]. Therefore, since overall expression of all TSFM isoforms and of the four reference sequence isoforms (TSFM WT) was not significantly altered by genotype, we conclude that the exon inclusion caused by the SNP is unlikely to be of functional significance.

The genotype-specific splicing changes of the GSDMB gene may be of more potential importance. Variant rs11078928, an A > G change in the acceptor splice site of intron 5 of the GSDMB gene, is associated with two separate alternative splicing events. Firstly the major (A) allele of rs11078928 is associated with exon skipping, resulting in a splice product missing exons 5-8 (referred to as GSDMB NV), whereas this does not occur in individuals carrying the minor $(G)$ allele. The cause of the deletion is not easy to ascertain, but could be attributable to changes in the RNA secondary structure induced by this SNP, which could alter binding of the splicing machinery (Figure 5). It is also possible that another SNP or SNPs in linkage disequilibrium (LD) with rs11078928 may be causing this splicing alteration. Although WT GSDMB and total GSDMB expression was decreased in those carrying the minor allele, these changes did not reach statistical significance, indicating that the disease susceptibility may be attributable to a gain of function effect from the novel transcript, rather than the amount of the GSDMB transcript pool (Figure 3). It is difficult however to speculate as to the effect of this large deletion on GSDMB protein function, since little is known about the structural function of this protein. Secondly, we identified that subjects carrying the minor (G) allele of rs11078928 exhibit skipping of exon 6 of the gene, with homozygotes for the minor allele
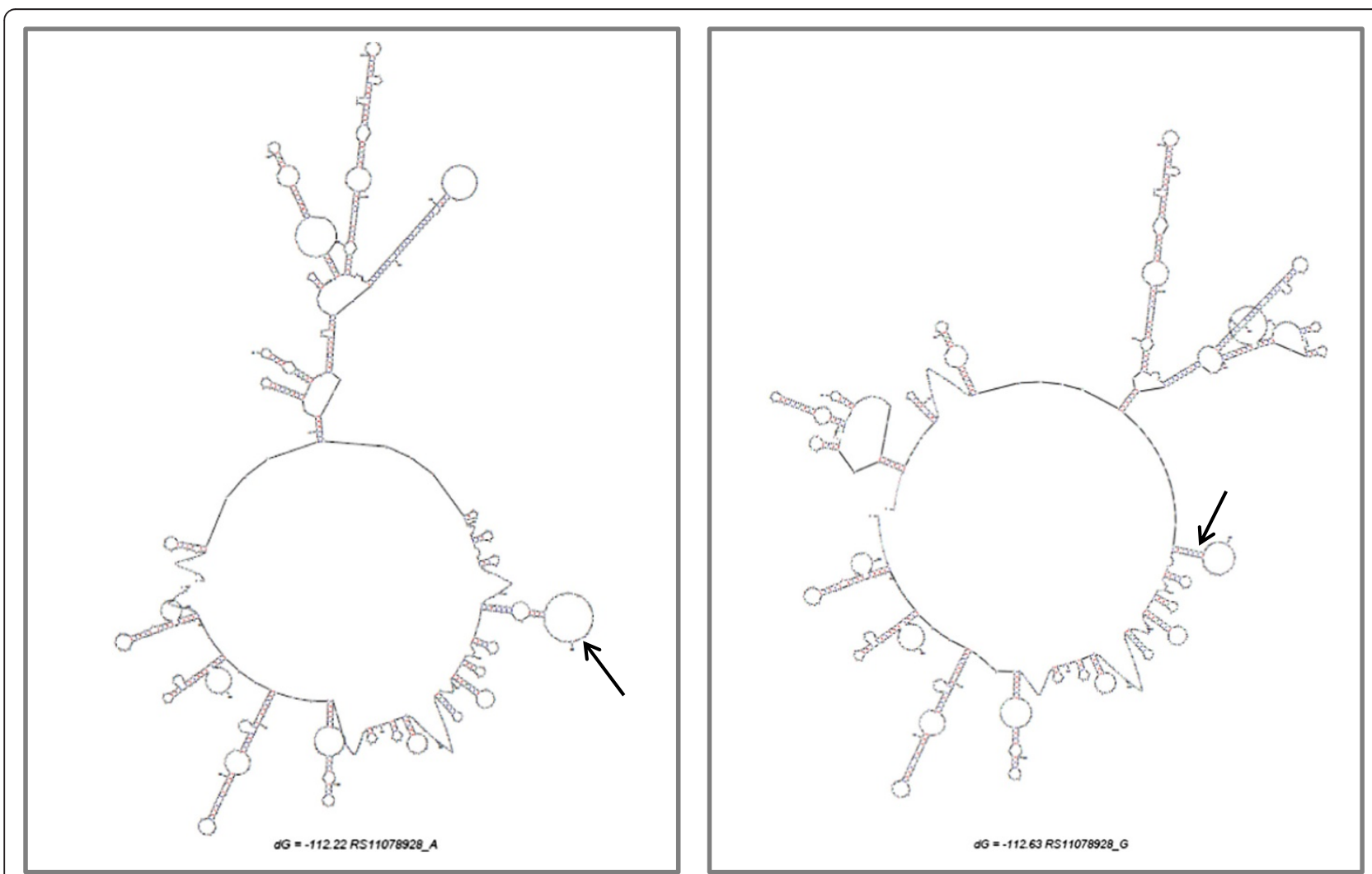

Figure 5 Showing the secondary structure change with the major A allele (right) and the minor allele G (left), as predicted by Mfold (http://mfold.rna.albany.edu/?q=mfold). The arrows indicate the position of the variant rs11078928 in the GSDMB transcript sequence. 
expressing very little full length product (transcripts containing exon 6).

Taken together, these changes indicate that whilst individuals homozygous for the major (A) allele do express the $\triangle 5-8$ deleted GSDMB product, they also express the full length exon 6-containing isoforms. Conversely, individuals homozygous for the minor $(G)$ allele do not express either the deleted GSDMB product or the full length product (isoforms containing exon 6), as reflected in their lower overall expression. This may suggest some functional importance of the region of the GSDMB protein encoded by exon 6 , but there is very little information on the nature of this region in the current literature.

GSDMB encodes for the protein Gasdermin B, which is a member of the gasdermin-domain containing protein family. Although the exact function of GSDMB remains unclear, members of the gasdermin-domain containing family have roles in epithelial cell apoptosis and in maintaining a differentiated state of epithelial cells [20]. GSDMB has also been shown to be important in cancer pathogenesis, with alternative splicing of the gene being involved in gastrointestinal and hepatic cancers [21]. The GSDMB gene contains several conserved amino acid sequences in the $\mathrm{N}$ and $\mathrm{C}$ terminal regions as well as several conserved leucine-rich motifs throughout the sequence [22]. The deletion product described here, GSDMB NV, is in frame so would not be subject to nonsense-mediated decay and could plausibly be translated into the protein.

Variant rs11078928 is located on chromosome 17q1221 , in an area which was identified in 2007 to be associated with asthma susceptibility (rs7216389), as well as susceptibility to developing several autoimmune diseases, including ulcerative colitis (rs2872507), Crohn's disease (rs2872507), type 1 diabetes (rs2290400) and primary biliary cirrhosis (rs9303277) (see Table 1) [23]. It is interesting to note that the risk alleles for allergic disease susceptibility are the alternative to that for autoimmune disease susceptibility, indicating opposite effects of the variants on pathogenesis of these diseases [24]. The minor allele for rs11078928, increases risk for developing these autoimmune traits (risk allele for type 1 diabetes not known) and confers a decreased risk to developing asthma. This association has been replicated several times by independent groups and in different populations, and there has been much debate over potential mechanisms of action and which is the causal gene(s) in that locus.

GSDMB, ORMDL3 (ORM1-like 3) and ZPBP2 (zona pellucida binding protein 2) have all been considered good candidates, as they are all expression quantitative trait loci, with opposite directions of expression seen with genotype for ORMDL3/GSDMB and ZPBP2. At present, the best functional evidence of the mechanism by which the 17q12-21 locus may alter asthma and autoimmune disease susceptibility comes from two studies by the same group, who identified a $5.3 \mathrm{~kb}$ region overlapping the $Z P B P 2$ gene, which is associated with several gene regulatory marks, including allele-specific chromatin interactions, DNA methylation and promoter activity $[20,25]$. It has been hypothesised that this regulatory region may be involved in long-range chromatin interactions and may influence the expression of any one or all of the genes GSDMB, ORMDL3 and ZPBP2 [20,25].

$Z P B P 2$ has a well-known role in fertilisation and male fertility, and it has been proposed that this gene may play a role in influencing the prevalence of asthma in the population [25]. ORMDL3 has so far been thought to be the most promising candidate at this locus, since it has a role in mediating inflammation, and also has expression in bronchial epithelial cells, where slightly higher ORMDL3 expression was found in individuals with asthma compared to controls [26,27]. GSDMB shows a diverse expression pattern in tissues, including expression in the lung, liver, intestine and colon, but with very low expression in bronchial epithelial cells. However, it has been suggested that the effect of the chromosome 17q12-21 locus on multiple diseases may reflect a more direct role on immune function rather than any tissue-specific effect $[20,28]$. The expression of GSDMB in the thymus and CD8+ and CD4+ T-cells would increase the plausibility of this inference, especially given the involvement of type 1 and type $2 \mathrm{im}-$ mune responses in autoimmune and allergic disease respectively [20,29].

Although genome-wide analysis of transcript isoform expression in the CEU HapMap population found no differences in the expression of GSDMB isoform ratios associated with the asthma haplotype in LCLs (lymphoblastoid cell lines) [30], the splicing of GSDMB has been shown to be associated with genotype of the related variant rs7216389 in brain and in peripheral blood mononucleated cells [31]. rs7216389 is in the same LD block as rs11078928 ( $r^{2} 0.905$, D'1.0), and our data thus suggest the effect on splicing noted in this, and in our study may be an effect of the splice site SNP rs11078928. Interestingly Heinzen et al. found an overrepresentation of autoimmune traits associated with splicing quantitative trait loci (sQTLs), which could indicate the importance of splicing differences in these diseases [31]. Finally, LCLs may not offer a realistic representation of regulation of in vivo gene expression [32], explaining the differences in GSDMB splicing patterns seen in primary cell types and LCLs.

These data are of course preliminary in nature, and require further work to assess the potential consequences of a reduction in GSDMB expression, or an alteration to the pool of transcripts expressed from the GSDMB gene 
to the function of tissues involved in immune function and inflammation. Future studies should focus on the effect of alterations to the GSDMB isoform pool on factors such as the inflammatory milieu in other appropriate cell types to prove causality and further define mechanism. It remains to be seen also if GSDMB splicing changes are also noted in individuals with asthma and autoimmune diseases.

\section{Conclusions}

To conclude, we have demonstrated that a proportion of genetic variants identified as susceptibility loci for inflammatory or autoimmune disease may act by disrupting the native splicing patterns of their host genes. Using bioinformatics to identify variants likely to interfere with splicing patterns, followed by functional evaluation in whole blood, we have demonstrated alterations in the splicing of the GSDMB and TSFM genes, which is associated with genotype at rs11078928 and rs2014886 respectively. Although the exon inclusion caused by the variant in TSFM is unlikely to have any functional significance, our data suggest that rs11078928 is associated with the production of a novel GSDMB transcript lacking an internal segment, together with a change in the ratio of some known isoforms. This is predicted to result in an almost complete lack of full length GSDMB mRNA in individuals homozygous for the minor allele. Although the functional significance of these changes remains to be determined, our study provides further evidence that GSDMB is a promising candidate gene at the 17q12-21 locus, in altering susceptibility to various autoimmune diseases, and asthma.

\section{Methods}

\section{Identifying candidate genes for splicing analysis}

We searched the National Institutes of Health (NIH) GWAS catalogue (http://www.genome.gov/gwastudies) [5] to identify SNPs associated with known autoimmune diseases at genome wide significance $\left(P<5 \times 10^{-8}\right)$. We focussed on inflammatory and autoimmune traits as we had access to an appropriate tissue, i.e. lymphocyte RNA, to assess splicing. We defined autoimmune diseases/ inflammatory traits as those described in the literature as 'autoimmune disease' or 'chronic inflammatory disease'. Next we identified proxy SNPs to these candidates that had $r^{2}>0.8$, and located within $500 \mathrm{~kb}$ of the index variant, using the SNAP Proxy Search tool (http://www. broadinstitute.org/mpg/snap/ldsearch.php) [33]. The functional consequence of each SNP on transcription was then identified using the BioMart function of Ensembl (http://www.ensembl.org) (Homo sapiens Variation (dbSNP build 135; ENSEMBL). SNPs labelled as 'Splice Site' or 'Essential Splice Site' were identified and these SNPs were analysed in silico using ESE Finder [34,35], Alamut Mutation Interpretation Software (Interactive Biosoftware, Rouen,
France), and Flybase from the Berkeley Drosophila Genome Project (http://www.fruitfly.org/seq_tools/splice.html) [36], in order to predict how likely a splicing change would occur as a result of the SNP (Figure 1 and Table 1). Variants demonstrating good evidence of the capacity to modify splicing patterns were prioritised for further transcriptomic analysis in mRNA derived from whole blood samples from subjects of defined genotype.

\section{Assessment of splice site enrichment for SNPs associated with inflammatory or autoimmune diseases}

To determine the likelihood that SNPs associated with autoimmune or inflammatory phenotypes were located in elements responsible for splice site choice by chance alone, we selected 1000 sets of 338 random SNPs matched on allele frequency $( \pm 5 \%)$ and gene proximity $( \pm 10 \mathrm{~kb})$. For each set we retrieved all variants in linkage disequilibrium $\left(\mathrm{r}^{2}>0.8\right.$ in 1000 Genomes Phase 1 data in Europeans) and used them to query the BioMart database using the 'biomaRt' package. In each set we calculated the proportion of SNPs identified by BioMart as 'splice_region_variant'. Finally, we compared the proportion of splice site variants in 338 autoimmune disease SNPs with proportions identified in 1000 sets of matched proxies and observed enrichment for splice site variants by one tailed $t$-test.

\section{Cohort information and sample collection}

The samples used were from the Exeter 10,000 cohort (http://www.exeter.crf.nihr.ac.uk/). GSDMB and TSFM total expression, expression of wild-type (WT) and novel $(\mathrm{NV})$ transcripts, as well as GSDMB isoform specific expression, were compared between 8-10 individuals homozygous for the major alleles, $8-10$ heterozygous individuals, and 5-8 individuals homozygous for the minor alleles. The individuals included in the cohort were of mixed gender and were of predominantly White British origin. $20 \%$ of the GSDMB WT, NV and isoform-specific expression cohort had diagnosed autoimmune disease (AA $\mathrm{n}=2$, AG $\mathrm{n}=2$ and $\mathrm{GG} \mathrm{n}=2$ ), as had $23 \%$ of the GSDMB total expression cohort (AA $\mathrm{n}=3, \mathrm{AG} \mathrm{n}=2$ and $\mathrm{GG} \mathrm{n}=0$ ) and $21 \%$ of the TSHM cohort $(\mathrm{CC} n=3$, CT $n=1$ and TT $n=$ 1). The median ages were as follows: GSDMB WT, NV and isoform-specific expression: 62 (AA $n=10) 61$ (AG n =10) $60(\mathrm{GG} \mathrm{n}=5)$; GSDMB total expression (expression of WT and alternative transcripts) $60(\mathrm{AA} \mathrm{n}=8) 56$ (AG n =8) 54 (GG $\mathrm{n}=6)$; TSFM WT, NV and total expression $46(\mathrm{CC} \mathrm{n}=8) 63(\mathrm{CT} \mathrm{n}=8) 37(\mathrm{TT} \mathrm{n}=8)$. The median ages between genotypes were compared using the Kruskal-Wallis $\mathrm{H}$ test and did not reach statistical significance $(\mathrm{P}<0.05)$ in the case of GSDMB. However, when comparing the median ages for the TSFM cohort, the differences between the groups of each genotype did reach statistical significance $(P=0.04)$. The median BMIs 
were as follows: GSDMB WT, NV, isoform-specific expression: 27 (AA) 25 (AG) 26 (GG); GSDMB total expression 25 (AA) 26 (AG) 25 (GG); TSFM WT, NV and total expression 25 (CC) 29 (CT) 22 (TT). The median BMIs were compared using the Kruskal-Wallis $\mathrm{H}$ test and did not reach statistical significant for any of the groups. Median white blood cell counts were compared using Kruskal-Wallis $\mathrm{H}$ test and did not reach statistical significance across any of the groups. Data and statistics for these parameters are given in Additional file 4. Current medication and past medical history were known for the donors. $2.5 \mathrm{ml}$ peripheral blood specimens were collected using PAXgene technology [37] and extracted using the PAXgene Blood mRNA kit (Qiagen, Crawley, UK) according to the manufacturer's instructions. Research was carried out in accordance with the Helsinki Declaration, and ethical approval was granted by the Bristol Regional Ethics Committee (study number 09/ H0106/75). Written informed consent was obtained for all participants.

\section{SNP genotyping}

PCR primers were designed to the area surrounding the SNP. The primer sequences were as follows: GSDMB forward ( $5^{\prime}-$ GGTGCGTCTTACCACATCCT-3'), GSDMB reverse (5'-G GGACTGGAGAAAGGGAACT-3'); TSFM forward (5'-GG CGAAACCCCATCTCTACT-3'), TSFM reverse (5'- CCCC CACACTGTCTGACTTT-3'); GCKR forward (5' - CCCTCC CCTTCTCCTAGACA-3'), GCKR reverse (5'- GCTGATG ATGGAGGGAAAGA-3'); $D L D$ forward (5'- CCTGAAAT AGATTTCCCTGACA-3'), DLD reverse (5'- GCCATCAGC TTTCGTAGCAG-3'); FGFR1OP forward (5'- GAAGGTT TTTGAGGGGGTAAA-3'), FGFR1OP reverse (5' - TTTCCC TCTGGTGACTTTGG-3'); STAT2 forward (5' - CACAGA CTCTGGTGGAGCAA-3'), STAT2 reverse (5'- TGCAGTT CCTCTGTCACACC-3'); ATXN2L forward (5' - TGGCCAG AAGAAGGGATAGA-3'), ATXN2L reverse (5'-AGATTCT GCTGTGGCTGTCC-3'); CLEC2D forward (5'-GGTGCCA CTTAAAAAGTTATTGG-3'), CLEC2D reverse (5'- AGTG TGTGGAATGGTTGCTG-3'). 50 ng DNA from the peripheral blood of healthy controls was amplified by PCR using MegaMix Royal (Cambio, Cambridge, UK) under the following conditions: $95^{\circ} \mathrm{C}$ for $5 \mathrm{~min}$; followed by 40 cycles of: $95^{\circ} \mathrm{C}$ for $30 \mathrm{~s}, 60^{\circ} \mathrm{C}$ for $1 \mathrm{~min}$, $72^{\circ} \mathrm{C}$ for $1 \mathrm{~min}$; followed by $72^{\circ} \mathrm{C}$ for $10 \mathrm{~min}$. PCR products were then sequenced to confirm genotype. Sequence analysis was carried out using the Mutation Surveyor software package (SoftGenetics, Pennsylvania, USA).

\section{RT-PCR and band isolation to identify novel splice products}

PCR primers were designed to at least 2 exons either side of the SNP of interest. The primer sequences were as follows:
GSDMB forward (5' - ACCCTTTTCATTCCGATCAA-3'), GSDMB reverse (5' - AAGTCCAGAATGGCTTTTGC-3'); TSFM forward (5'- GGTGTTTATCGCGGCTAGAG -3'), TSFM reverse ( $5^{\prime}$ - CAGAGGGTTGATCCTTTAGGG -3'); TSFM NV forward (5'- GACCTCAAACAGACGGAG TCTTGCT-3'), TSFM NV reverse (5' - TCTTTGGTCTTC CTCCCTTGGAGC-3'), GCKR forward (5'- GGCTTTCT CATTGGTGATCACAGTGA-3'), GCKR reverse (5' - AGC TTGGAGTTGCTAATCCGAAGGT-3'); DLD forward ( $5^{\prime}-$ CTTGGTGGAACATGCTTGAA-3'), DLD reverse (5' - CC ATCTGACTTCTTGGTAGCAC-3'), FGFR1OP forward (5' - TCCTTTAGTTAATGAGAGCCTGAAA-3'), FGFR1OP reverse (5' CAGACTTCCTGCTTGCTTCC-3'); STAT 2 forward ( $5^{\prime}$ - TCCTCCTCAATTACAAGGCTTC-3'), STAT2 reverse (5' - TGCTCAGCTGGTCTGAGTTG-3'); ATXN2L forward (5' - CCAAGCCCTTTATGCCACT-3'), $A T X N 2 L$ reverse (5'- GAAGCTGCTCTGAGGGGACT-3'); CLEC2D forward (5'- AACCCAGGTTGTCTGCATTC-3'), CLEC2D reverse (5' - TTCAGTACCATTTATCCATTTCC A-3'). 100 ng peripheral blood RNA from individuals of known genotype, was reverse transcribed using SuperScript ${ }^{\circ}$ III First-Strand Synthesis System following the protocol as recommended by the manufacturer (Invitrogen by Life Technologies, Foster City, USA). The cDNA was then amplified by PCR using MegaMix Royal (Cambio, Cambridge, UK) under the following conditions: $95^{\circ} \mathrm{C}$ for $5 \mathrm{~min}$; followed by 30 cycles of: $95^{\circ} \mathrm{C}$ for $30 \mathrm{~s}, 60^{\circ} \mathrm{C}$ for $1 \mathrm{~min}, 72^{\circ} \mathrm{C}$ for $1 \mathrm{~min}$; followed by $72^{\circ} \mathrm{C}$ for $10 \mathrm{~min}$, and the products were separated on a $2 \%$ agarose gel. Novel bands were identified, isolated and amplified using the respective PCR primers and conditions already described. The amplification products were then sequenced to confirm identity and characterise any unusual products.

\section{Real-time PCR to quantify relative expression of isoforms by genotype}

Real-time PCR primers were designed to the reference sequence (wild-type; WT) and any novel (novel; NV) transcripts. Where alternatively expressed isoforms were present in the region of interest, inventoried Taqman Assays were purchased (Life Technologies, Foster City, USA), information for which is shown in Additional file 2. Assays for total expression of all wild-type transcripts as well as the novel transcripts were also purchased. Primer and probe information for Custom Taqman Assays are given in Additional file 5, and their relative positions are indicated in Figures 2 and 3. RNA was reverse transcribed as described previously, and real-time quantitative PCR was performed using the ABI Prism 7900HT platform. RNA from 5-10 individuals of each genotype was amplified by this method (10X heterozygotes, 10X homozygotes for the major allele, 5-10X homozygotes for the minor allele). Results were analysed using the 
Comparative Ct method [38] to identify genotypespecific alternations in transcript levels.

\section{Assessment of secondary structure change}

Pre-spliced hnRNA secondary structure was analysed using MFOLD software (http://www.bioinfo.rpi.edu/applications/ mfold/), under default settings [39,40].

\section{Statistical analysis}

Differences between the expression patterns of normal and aberrant transcripts according to genotype were assessed by the Kruskal-Wallis $\mathrm{H}$ test. Non-parametric statistics were necessary due to the relatively small sample numbers and the expected non-normality of data.

\section{Additional files}

Additional file 1: (Additional data file 1.ppt) is a figure showing the difference in nucleotide sequence between GSDMB WT and GSDMB NV transcript sequences. The title of the figure is "Electropherogram showing changes to the GSDMB transcript at the sequence level".

Additional file 2: (Additional data file 2.csv) is a table showing the Taqman Assay information, including Assay IDs (Applied Biosystems, Foster City, USA) along with information about the transcript(s) that the assay will detect. The title of the table is "Taqman Assay information".

Additional file 3: (Additional data file 3.ppt) is a figure showing the difference in nucleotide sequence between TSFM WT and TSFM NV transcript sequences. The title of the figure is "Electropherogram showing the changes to the TSFM transcript at the sequence level".

Additional file 4: (Additional data file 4.docx) is a table giving the age, BMI, white blood count, Platelet count and haemoglobin measurements for study participants.

Additional file 5: (Additional data file 5.csv) is a table listing the Custom Taqman Assay information, including primer and reporter information, and transcripts detected by the Custom Taqman Assays, provided by Applied Biosystems (Foster City, USA). The title of the table is "Custom Taqman Assay information for GSDMB and TSFM".

\section{Abbreviations}

SNP: Single nucleotide polymorphism; GWAS: Genome Wide Association Studies; LD: Linkage disequilibrium; GSDMB: Gasdermin B; TSFM: Ts translation elongation factor, mitochondrial; WT: Wild-type; MT: Mutant; NV: Novel variant; LCL: Lymphoblastoid cell line.

\section{Competing interests}

The authors declare that they have no competing interests.

\section{Authors' contributions}

FSM carried out all experimental and statistical analyses, and was responsible for drafting the manuscript. JML participated in design of the study, and participated in its coordination and implementation. ARW and MT participated in the bioinformatic analyses. DP carried out the assessment of splice site enrichment for SNPs associated with inflammatory and autoimmune diseases. AM and TF contributed to the study, and participated in its design and coordination. LWH managed the study, oversaw final approval of the manuscript and contributed funding. All authors read and approved the final manuscript.

\section{Acknowledgements}

The authors wish to acknowledge the clinicians who assisted in the collection of the samples and Diane Jarvis for extraction of the samples. This work was supported by a charitable donation from the Mendip Golf Club to [L.W.H]. This project was supported by the NIHR Exeter Clinical Research Facility. This project does not necessarily reflect the views of the NIHR Exeter Clinical Research Facility, the NHS or the Department of Health.
Author details

'RNA mediated mechanisms of disease group, University of Exeter Medical School, EX2 5DW Exeter, UK. ${ }^{2}$ Genetics of Complex Traits, University of Exeter Medical School, EX1 2LU Exeter, UK.

Received: 8 April 2013 Accepted: 16 September 2013

Published: 17 September 2013

\section{References}

1. Bowcock AM: Genomics: guilt by association. Nature 2007, 447:645-646.

2. Wellcome Trust Case Control Consortium: Genome-wide association study of 14,000 cases of seven common diseases and 3,000 shared controls. Nature 2007, 447:661-678.

3. Lettre G, Rioux JD: Autoimmune diseases: insights from genome-wide association studies. Hum Mol Genet 2008, 17:R116-R121.

4. Brest P, Lapaquette P, Souidi M, Lebrigand K, Cesaro A, Vouret-Craviari V, Mari B, Barbry P, Mosnier JF, Hebuterne X, et al: A synonymous variant in IRGM alters a binding site for miR-196 and causes deregulation of IRGMdependent xenophagy in Crohn's disease. Nat Genet 2011, 43:242-245.

5. Hindorff LA, Sethupathy P, Junkins HA, Ramos EM, Mehta JP, Collins FS, Manolio TA: Potential etiologic and functional implications of genomewide association loci for human diseases and traits. Proc Natl Acad Sci U S A 2009, 106:9362-9367.

6. Ernst J, Kheradpour P, Mikkelsen TS, Shoresh N, Ward LD, Epstein CB, Zhang X Wang L, Issner R, Coyne M, et al: Mapping and analysis of chromatin state dynamics in nine human cell types. Nature 2011, 473:43-49.

7. ENCODE Project Consortium: An integrated encyclopedia of DNA elements in the human genome. Nature 2012, 489:57-74

8. Thurman RE, Rynes E, Humbert R, Vierstra J, Maurano MT, Haugen E, Sheffield NC, Stergachis AB, Wang H, Vernot B, et al: The accessible chromatin landscape of the human genome. Nature 2012, 489:75-82.

9. Neph S, Vierstra J, Stergachis AB, Reynolds AP, Haugen E, Vernot B, Thurman $R E$, John $S$, Sandstrom R, Johnson AK, et al: An expansive human regulatory lexicon encoded in transcription factor footprints. Nature 2012, 489:83-90.

10. Gerstein MB, Kundaje A, Hariharan M, Landt SG, Yan K-K, Cheng C, Mu XJ, Khurana E, Rozowsky J, Alexander R, et al: Architecture of the human regulatory network derived from ENCODE data. Nature 2012, 489:91-100.

11. Djebali S, Davis CA, Merkel A, Dobin A, Lassmann T, Mortazavi A, Tanzer A, Lagarde J, Lin W, Schlesinger F, et al: Landscape of transcription in human cells. Nature 2012, 489:101-108.

12. Sanyal $A$, Lajoie $B R$, Jain $G$, Dekker J: The long-range interaction landscape of gene promoters. Nature 2012, 489:109-113.

13. Cartegni $L$, Chew SL, Krainer AR: Listening to silence and understanding nonsense: exonic mutations that affect splicing. Nat Rev Genet 2002, 3:285-298.

14. Staley JP, Woolford JL Jr: Assembly of ribosomes and spliceosomes: complex ribonucleoprotein machines. Curr Opin Cell Biol 2009, 21:109-118.

15. Benson DA, Karsch-Mizrachi I, Lipman DJ, Ostell J, Wheeler DL: GenBank: update. Nucleic Acids Res 2004, 32:D23-D26.

16. Blencowe BJ: Alternative splicing: new insights from global analyses. Cell 2006, 126:37-47.

17. Frischmeyer PA, Dietz HC: Nonsense-mediated mRNA decay in health and disease. Hum Mol Genet 1999, 8:1893-1900.

18. Handel AE, Handunnetthi L, Berlanga AJ, Watson CT, Morahan JM, Ramagopalan SV: The effect of single nucleotide polymorphisms from genome wide association studies in multiple sclerosis on gene expression. PLoS One 2010, 5:e10142.

19. Alcina A, Fedetz M, Fernandez O, Saiz A, Izquierdo G, Lucas M, Leyva L, Garcia-Leon JA, Abad-Grau Mdel M, Alloza I, et al: Identification of a functional variant in the KIF5A-CYP27B1-METTL1-FAM119B locus associated with multiple sclerosis. J Med Genet 2013, 50:25-33.

20. Verlaan DJ, Berlivet $S$, Hunninghake GM, Madore AM, Lariviere M, Moussette $S$, Grundberg E, Kwan T, Ouimet M, Ge B, et al: Allele-Specific Chromatin Remodeling in the ZPBP2/GSDMB/ORMDL3 Locus Associated with the Risk of Asthma and Autoimmune Disease. Am J Hum Genet 2009, 85:377-393.

21. Carl-Mcgrath S, Schneider-Stock R, Ebert M, Rocken C: Differential expression and localisation of gasdermin-like (GSDML), a novel member of the cancer-associated GSDMDC protein family, in neoplastic and nonneoplastic gastric, hepatic, and colon tissues. Pathology 2008, 40:13-24. 
22. Tamura M, Tanaka S, Fujii T, Aoki A, Komiyama H, Ezawa K, Sumiyama K, Sagai T, Shiroishi T: Members of a novel gene family, Gsdm, are expressed exclusively in the epithelium of the skin and gastrointestinal tract in a highly tissue-specific manner. Genomics 2007, 89:618-629.

23. Moffatt MF, Kabesch M, Liang LM, Dixon AL, Strachan D, Heath S, Depner M, Von Berg A, Bufe A, Rietschel E, et al: Genetic variants regulating ORMDL3 expression contribute to the risk of childhood asthma. Nature 2007, 448:470-U475.

24. Li X, Ampleford EJ, Howard TD, Moore WC, Torgerson DG, Li H, Busse WW, Castro M, Erzurum SC, Israel E, et al: Genome-wide association studies of asthma indicate opposite immunopathogenesis direction from autoimmune diseases. J Allergy Clin Immunol 2012, 130:861-868.

25. Berlivet S, Moussette S, Ouimet M, Verlaan DJ, Koka V, Al Tuwaijri A, Kwan T, Sinnett D, Pastinen T, Naumova AK: Interaction between genetic and epigenetic variation defines gene expression patterns at the asthmaassociated locus 17q12-q21 in lymphoblastoid cell lines. Hum Genet 2012, 131:1161-1171.

26. Cantero-Recasens G, Fandos C, Rubio-Moscardo F, Valverde MA, Vicente R: The asthma-associated ORMDL3 gene product regulates endoplasmic reticulum-mediated calcium signaling and cellular stress. Hum Mol Genet 2010, 19:111-121.

27. Bochkov YA, Hanson KM, Keles S, Brockman-Schneider RA, Jarjour NN, Gern JE: Rhinovirus-induced modulation of gene expression in bronchial epithelial cells from subjects with asthma. Mucosal Immunol 2010, 3:69-80.

28. Su Al, Wiltshire T, Batalov S, Lapp H, Ching KA, Block D, Zhang J, Soden R, Hayakawa M, Kreiman G, et al: A gene atlas of the mouse and human proteinencoding transcriptomes. Proc Natl Acad Sci U S A 2004, 101:6062-6067.

29. Rabin RL, Levinson Al: The nexus between atopic disease and autoimmunity: a review of the epidemiological and mechanistic literature. Clin Exp Immunol 2008, 153:19-30.

30. Kwan T, Benovoy D, Dias C, Gurd S, Provencher C, Beaulieu P, Hudson TJ, Sladek R, Majewski J: Genome-wide analysis of transcript isoform variation in humans. Nat Genet 2008, 40:225-231.

31. Heinzen EL, Ge DL, Cronin KD, Maia JM, Shianna KV, Gabriel WN, WelshBohmer KA, Hulette CM, Denny TN, Goldstein DB: Tissue-Specific Genetic Control of Splicing: Implications for the Study of Complex Traits. PLOS Biol 2008, 6:2869-2879.

32. Min JL, Taylor JM, Richards JB, Watts T, Pettersson FH, Broxholme J, Ahmad KR, Surdulescu GL, Lowy E, Gieger C, et al: The use of genome-wide eQTL associations in lymphoblastoid cell lines to identify novel genetic pathways involved in complex traits. PLoS One 2011, 6:e22070.

33. Johnson AD, Handsaker RE, Pulit SL, Nizzari MM, O'Donnell CJ, De Bakker PI: SNAP: a web-based tool for identification and annotation of proxy SNPs using HapMap. Bioinformatics Oxford, England 2008, 24:2938-2939.

34. Smith PJ, Zhang C, Wang J, Chew SL, Zhang MQ, Krainer AR: An increased specificity score matrix for the prediction of SF2/ASF-specific exonic splicing enhancers. Hum Mol Genet 2006, 15:2490-2508.

35. Cartegni L, Wang J, Zhu Z, Zhang MQ, Krainer AR: ESEfinder: A web resource to identify exonic splicing enhancers. Nucleic Acids Res 2003, 31:3568-3571.

36. Reese MG, Eeckman FH, Kulp D, Haussler D: Improved splice site detection in Genie. Journal of computational biology: a journal of computational molecular cell biology 1997, 4:311-323.

37. Debey-Pascher S, Eggle D, Schultze JL: RNA stabilization of peripheral blood and profiling by bead chip analysis. Methods Mol Biol 2009, 496:175-210.

38. Pfaffl MW: A new mathematical model for relative quantification in realtime RT-PCR. Nucleic Acids Res 2001, 29:e45.

39. Zuker M: Mfold web server for nucleic acid folding and hybridization prediction. Nucleic Acids Res 2003, 31:3406-3415.

40. Mathews DH, Sabina J, Zuker M, Turner DH: Expanded sequence dependence of thermodynamic parameters improves prediction of RNA secondary structure. J Mol Biol 1999, 288:911-940.

\section{doi:10.1186/1471-2164-14-627}

Cite this article as: Morrison et al:: The splice site variant rs 11078928

may be associated with a genotype-dependent alteration in expression of GSDMB transcripts. BMC Genomics 2013 14:627. 